\title{
UNIFORM CONTINUITY AND COMPACTNESS IN TOPOLOGICAL GROUPS
}

\author{
J. M. KISTER ${ }^{2}$
}

A topological group $G$ will be said to have property $U$ if every continuous real-valued function $f$ on $G$ is uniformly continuous (i.e. for every $\epsilon>0$ there is a neighborhood $N$ of the identity in $G$ such that if $x$ and $y$ are in $G$ with $x y^{-1}$ in $N$ then $\left.|f(x)-f(y)|<\epsilon\right)$. It is well known that any compact topological group has property $U$ and clearly any discrete group has property $U$. It is natural to consider whether the converse holds: is every topological group having property $U$ compact or discrete?

This note shows that the answer in general is in the negative, but that affirmative results in this direction hold even in uniform spaces, and if one assumes the group is locally compact then the converse is true.

In verifying some properties of the counterexamples it will be useful to have the following lemma concerning the Stone-Cech compactification $\beta(X)$ of $X$ for certain spaces.

Terminology, mainly, will be that in [1]. Spaces are Hausdorff.

Lemma. Let $\left\{K_{\alpha}\right\}_{\alpha \in A}$ be a collection of compact spaces and $G$ the Cartesian product. Let $0=\left(0_{\alpha}\right)$ be a point in $G$ and $H$ the subset of $G$ consisting of all points $p=\left(p_{\alpha}\right)$ such that $p_{\alpha} \neq 0_{\alpha}$ for at most countably many $\alpha$ 's. Then $\beta(H)=G$.

PRoof. Using the characterization of $\beta(H)$ in terms of extensions it suffices to show that every bounded continuous real-valued function $f$ on $H$ can be extended to $G$, since $H$ is clearly dense in $G$ and $G$ is compact.

First, $H$ is countably compact, since if $\left\{p_{i}\right\}$ is any countable sequence, then $p_{i \alpha} \neq 0_{\alpha}$ for at most a countable number of pairs $(i, \alpha)$. Let $\alpha_{1}, \alpha_{2}, \alpha_{3}, \cdots$ be all $\alpha^{\prime}$ 's that occur in such pairs. Then $\left\{p_{i}\right\}$ is contained in $F$, the subset of $H$ consisting of all points in $G$ whose $\alpha$ th coordinate is $0_{\alpha}$ for $\alpha \neq \alpha_{i}, i=1,2, \cdots$, and $F$ is closed in $G$, hence $\left\{p_{i}\right\}$ has a limit point in $F$.

Next, it will be shown that there exist a countable number of $\alpha$ 's, $\alpha_{1}, \alpha_{2}, \cdots$ so that if $p$ and $q$ are any two points in $H$ such that $p_{\alpha_{i}}=q_{\alpha_{i}}, i=1,2, \cdots$, then $f(p)=f(q)$. Suppose this is false.

Presented to the Society, April 17, 1959; received by the editors February 6, 1961.

${ }^{1}$ Supported by a fellowship from Gulf Research and Development Company.

2 The author wishes to express his gratitude to R. H. Bing for his encouragement. 
Let $p_{1}$ and $q_{1}$ be in $H$ and $f\left(p_{1}\right) \neq f\left(q_{1}\right)$, and let $A_{1}$ be all those $\alpha$ 's such that either $p_{1 \alpha} \neq 0_{\alpha}$ or $q_{1 \alpha} \neq 0_{\alpha}$.

Now let $p_{2}$ and $q_{2}$ be in $H, f\left(p_{2}\right) \neq f\left(q_{2}\right)$, and $p_{2 \alpha}=q_{2 \alpha}$ for all $\alpha$ in $A_{1}$. Let $A_{2}$ consist of $A_{1}$ together with all those $\alpha$ such that either $p_{2 \alpha} \neq 0_{\alpha}$ or $q_{2 \alpha} \neq 0_{\alpha}$.

This process is continued, via transfinite induction, as follows: Suppose $\beta$ is a countable ordinal and that $p_{\gamma}$ and $q_{\gamma}$, in $H$ have been found, for all $\gamma<\beta$, such that $f\left(p_{\gamma}\right) \neq f\left(q_{\gamma}\right)$, and $A_{\gamma}$ is a countable set which contains $A_{\delta}$, for every $\delta<\gamma$, as well as all those $\alpha$ such that either $p_{\gamma \alpha} \neq 0_{\alpha}$ or $q_{\gamma \alpha} \neq 0_{\alpha}$. Also suppose that $p_{\gamma \alpha}=q_{\gamma \alpha}$ for all $\alpha$ in $\mathrm{U}_{\delta<\gamma} A_{\varepsilon}$. Then, since $\beta$ is a countable ordinal, $\mathrm{U}_{\gamma<\beta} A_{\gamma}$ is countable and $p_{\beta}$ and $q_{\beta}$ may be found in $H$ such that $f\left(p_{\beta}\right) \neq f\left(q_{\beta}\right)$, and $p_{\beta \alpha}=q_{\beta \alpha}$ for all $\alpha$ in $\mathrm{U}_{\gamma<\beta} A_{\gamma} . A_{\beta}$ is defined to contain $\mathrm{U}_{\gamma<\beta} A_{\gamma}$ as well as those $\alpha$ such that either $p_{\beta \alpha} \neq 0_{\alpha}$ or $q_{\beta \alpha} \neq 0_{\alpha}$. In this way two transfinite sequences, $\left\{p_{\beta}\right\}$ and $\left\{q_{\beta}\right\}$, of length $\boldsymbol{\aleph}_{1}$, are obtained. If $d_{\beta}=\left|f\left(p_{\beta}\right)-f\left(q_{\beta}\right)\right|$ there is an $\epsilon>0$ and an uncountable set of countable ordinals $B$ such that $d_{\beta} \geqq \epsilon$ for all $\beta \in B$. Choose $\beta_{1}<\beta_{2}<\beta_{3}<\cdots$, a countable sequence in $B$. Since $H$ is countably compact $\left\{p_{\beta_{i}}\right\}$ has a limit point $r$. By construction $r$ is a limit point of $\left\{q_{\beta_{i}}\right\}$ and even stronger: if $N$ is a neighborhood of $r$ there exists an $i$ such that both $p_{\beta_{i}}$ and $q_{\beta_{i}}$ are in $N$. But then $f$ fails to be continuous at $r$, a contradiction.

Now $f$ can be extended to $f^{*}$ on $G$ as follows: let $\alpha_{1}, \alpha_{2}, \cdots$ be those $\alpha$ 's guaranteed by the preceding. If $p$ is in $G$ let $q$ be any point in $H$ such that $p_{\alpha_{i}}=q_{\alpha_{i}}, i=1,2, \cdots$. Define $f^{*}(p)=f(q) . f^{*}$ is clearly continuous, and the lemma is proved.

Examples of nondiscrete noncompact topological groups having property $U$. To get an example, $H$, which is countably compact, let $G$ be the Cartesian product of $\aleph_{1}$ compact nontrivial groups and $H$ the subgroup consisting of all elements having only a countable number of coordinates different from the identity. $H$ is countably compact, as was noted in the proof of the lemma; $H$ fails to be compact since it is not closed in $G$, and if $f$ is any continuous real function on $H$ it is bounded and by the lemma it can be extended to $G$. But since $G$ is compact the extension will be uniformly continuous on $G$, hence $f$ will be uniformly continuous on $H$.

To get another example, $K$, which is not countably compact, let $G$ be the Cartesian product of $\boldsymbol{\aleph}_{1}$ copies of the reals modulo 1 . Let $K$ be the subgroup of elements whose coordinates, except for a countable number, are all rational. Let $H$ be as in the previous example. $K$ is not countably compact, since if $\left\{r_{i}\right\}$ is any sequence of rationals converging to an irrational, each $r_{i}$ determines an element $p_{i}$ in $K$, all of whose coordinates are $r_{i}$ modulo 1 , and $\left\{p_{i}\right\}$ has no limit point 
in $K$. It is true that $K$ is pseudo-compact (real continuous functions on $K$ are all bounded), however, since $K$ contains $H$ as a dense subset and $H$ is countably compact. To see that $K$ has property $U$, one notes that if $f$ is a continuous real function on $K, f \mid H$ can be extended by the lemma to get $f^{*}$ on $G$, which will then be uniformly continuous, and $f^{*} \mid K=f$ is uniformly continuous on $K$.

A uniform space $(X, \mathcal{u})$ will be called uniformly locally bounded ${ }^{3}$ (ULB) provided there is an element $U$ of the uniformity $u$ with the property that for any $V$ in $\mathcal{u}$, there is an integer $n=n(V)$ such that for any $x$ in $X, U[x]$ is the union of at most $n$ sets $A$ in $X$ such that $A \times A \subset V$.

THEOREM. If $(X, \mathfrak{u})$ is a normal ULB uniform space on which all continuous real functions are uniformly continuous relative to $u$, and if $X$ has at most a finite number of isolated points then $X$ is countably compact.

Proof. If not, let $x_{1}, x_{2}, \ldots$ be a closed discrete set in $X$ and $U$ the element guaranteed by ULB. Find $N_{1}, N_{2}, \cdots$ such that $N_{i}$ is a neighborhood of $x_{i}$ contained in $U\left[x_{i}\right]$, and, using normality, $N_{i} \cap N_{j}$ $=\varnothing$ for $i \neq j$. Next, using normality again, obtain an open set $M$ such that each $x_{i}$ is in $M$ and $\mathrm{Cl} M \subset \bigcup_{i=1}^{\infty} N_{i}$. Let $M_{i}=M \cap N_{i}$. Note that $\mathrm{Cl}\left(\bigcup_{i=1}^{\infty} M_{i}\right)=\bigcup_{i=1}^{\infty}\left(\mathrm{Cl} M_{i}\right)$.

In $M_{i}$ choose distinct points $y_{i}^{1}, y_{i}^{2}, \cdots, y_{i}^{i}$, if possible, and define the function $f$ by $f\left(y_{i}^{J}\right)=j$ for all $i$ and $j \leqq i$. Since the set of all points of the form $y_{t}^{j}$ is closed, we can extend $f$ to a real continuous function defined on all of $X . f$ is not uniformly continuous, however. For let $V$ be an element of $\mathcal{u}$ such that if $A$ is a set in $X$ with $A \times A \subset V$ then $f(A)$ has diameter less than 1 . Now if $n=n(V)$ then $M_{n+1} \subset U\left[x_{n+1}\right]$ can be covered by at most $n$ sets $A$ where $A \times A \subset V$. Hence $f\left(M_{n+1}\right)$ containing $\{1,2, \cdots, n+1\}$ can be covered by $n$ sets of diameter less than 1 , which is impossible.

A topological group is locally bounded if it has a neighborhood of the identity which can be covered by finitely many translates of any other neighborhood of the identity. Clearly a locally bounded group, with its left uniformity, is a ULB uniform space.

CoROllaRY 1. A normal nondiscrete locally bounded topological group having property $U$ is countably compact.

Corollary 1 shows that the example $K$, previously considered, is

3 This is a local concept for uniform spaces of what is variously termed total boundedness or precompactness (cf. [1]) and is a consequence of that concept. 
not normal, since it is locally bounded, being the dense subgroup of a compact group.

CoRollary 2. A locally compact group with property $U$ is either discrete or compact.

Proof. A locally compact group is clearly locally bounded and paracompact $[1$, p. 172], hence normal, and compactness and countable compactness are equivalent in paracompact spaces.

In the nondiscrete case, the theorem generalizes a result of Samuel [2] to the effect that a normal space, all of whose uniform structures are precompact, is countably compact, since one chooses the uniform structure to be that which makes all real continuous functions uniformly continuous.

Question. Is eyery nondiscrete group having property $U$ pseudocompact?

\section{REFERENCES}

1. J. L. Kelley, General topology, Van Nostrand, New York, 1955.

2. P. Samuel, Ultrafilters and compactification of uniform spaces, Trans. Amer. Math. Soc. 64 (1948), 100-132.

UNIVERSITY OF Michigan 